\title{
Nonlinear Waveforms for Ion-Acoustic Waves in Weakly Relativistic Plasma of Warm Ion-Fluid and Isothermal Electrons
}

\author{
S. A. El-Wakil, Essam M. Abulwafa, E. K. El-Shewy, \\ H. G. Abdelwahed, and Hamdi M. Abd-El-Hamid \\ Theoretical Physics Group, Physics Department, Faculty of Science, Mansoura University, \\ Mansoura 35516, Egypt \\ Correspondence should be addressed to Essam M. Abulwafa, abulwafa@mans.edu.eg
}

Received 31 March 2012; Accepted 19 June 2012

Academic Editor: Wen Xiu Ma

Copyright (c) 2012 S. A. El-Wakil et al. This is an open access article distributed under the Creative Commons Attribution License, which permits unrestricted use, distribution, and reproduction in any medium, provided the original work is properly cited.

\begin{abstract}
The reductive perturbation method has been employed to derive the Korteweg-de Vries (KdV) equation for small- but finite-amplitude electrostatic ion-acoustic waves in weakly relativistic plasma consisting of warm ions and isothermal electrons. An algebraic method with computerized symbolic computation is applied in obtaining a series of exact solutions of the KdV equation. Numerical studies have been made using plasma parameters which reveal different solutions, that is, bell-shaped solitary pulses, rational pulses, and solutions with singularity at finite points, which called "blowup" solutions in addition to the propagation of an explosive pulses. The weakly relativistic effect is found to significantly change the basic properties (namely, the amplitude and the width) of the ion-acoustic waves. The result of the present investigation may be applicable to some plasma environments, such as ionosphere region.
\end{abstract}

\section{Introduction}

Nonlinear evolution equations are widely used as models to describe complex physical phenomena and have a significant role in several scientific and engineering fields [1,2]. The propagation of solitary waves is important as it describes characteristic nature of the interaction of the waves and the plasmas. In the case where the velocity of particles is much smaller than that of light, ion-acoustic waves present the nonrelativistic behaviors, but in the case where the velocity of particles approaches that of light, the relativistic effect becomes dominant [3]. Actually high-speed and energetic streaming ions with the energy from 0.1 to $100 \mathrm{MeV}$ are frequently observed in solar atmosphere and interplanetary space. Nevertheless, 


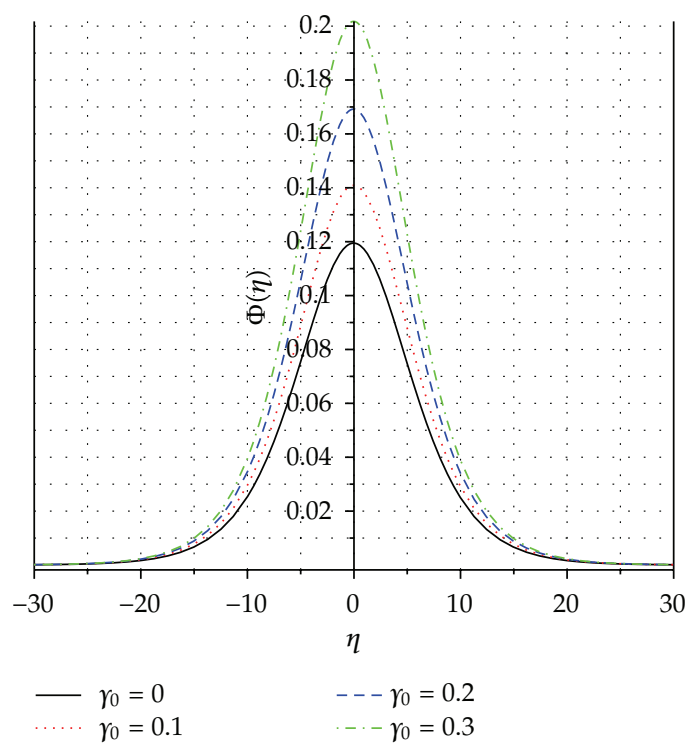

(a)

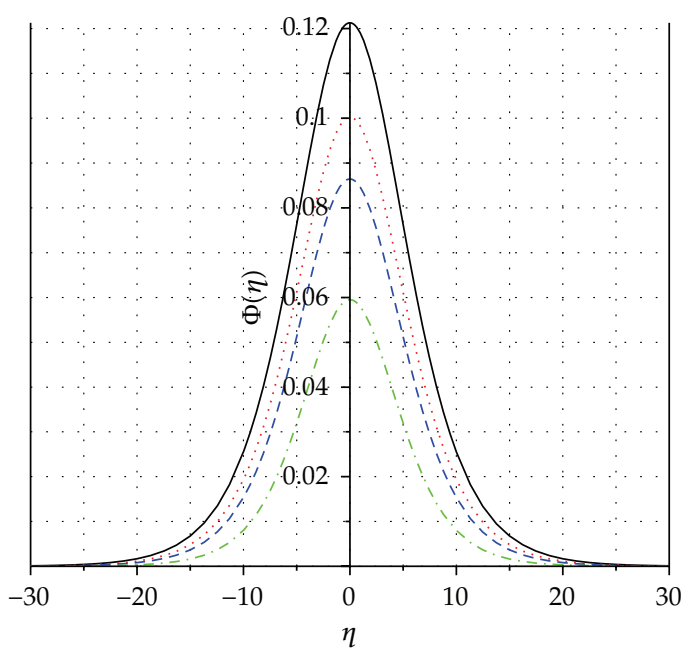

$\begin{aligned} \sigma & =0.001 \\ \sigma & =0.05\end{aligned}$

$---\sigma=0.1$

(b)

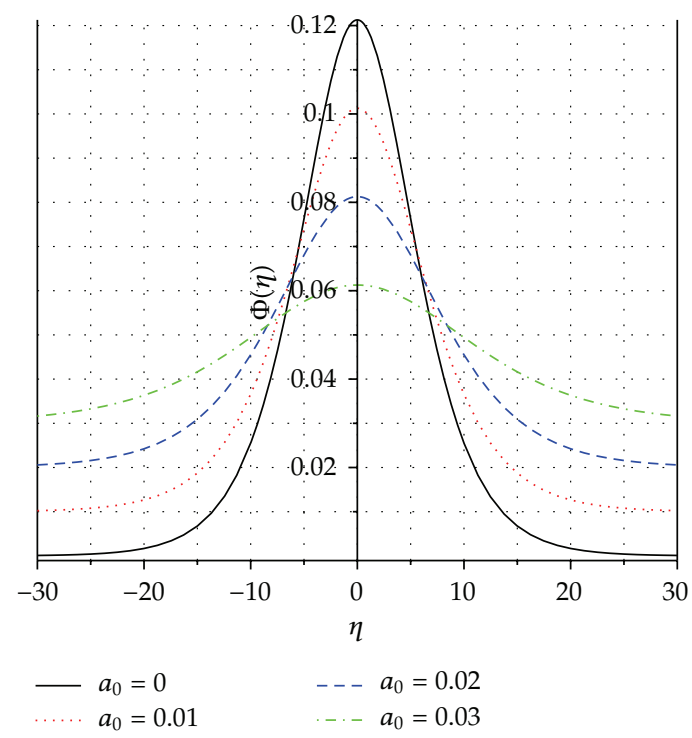

(c)

Figure 1: Profile of localized pulses for expression (4.3).

relativistic ion-acoustic waves have not been well investigated. When we assume that the ion energy depends only on the kinetic energy, such plasma ions have to attain very high velocity of relativistic order. Thus, by considering the weakly relativistic effect where the ion velocity is about $1 / 10$ of the velocity of light, we can describe the relativistic motion of such ions in the study of nonlinear interaction of the waves and the plasmas [4]. It appears that the weakly relativistic and ion temperature effects play an important role in energetic ion-acoustic waves propagating in interplanetary space $[5,6]$. 


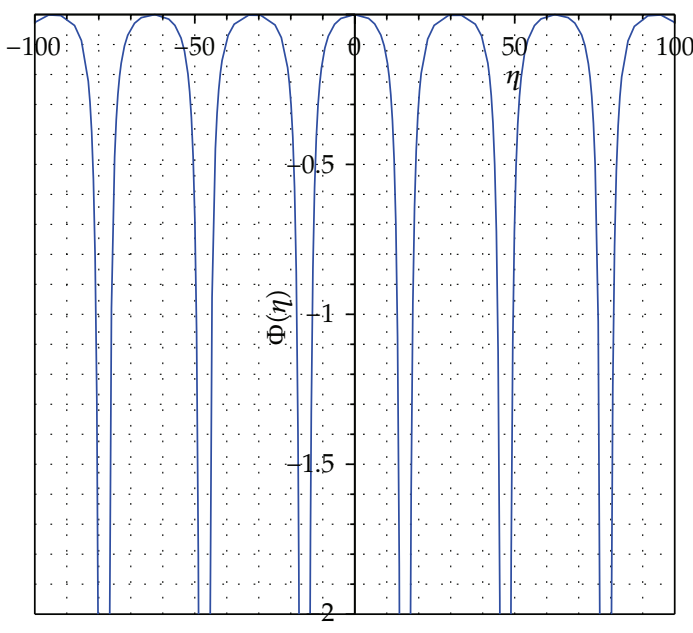

$-a_{0}=0$

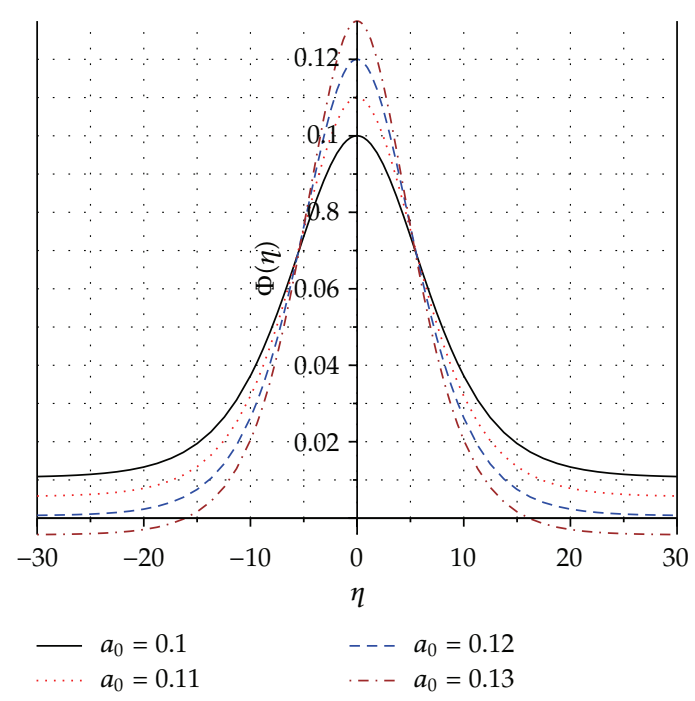

(b)

Figure 2: Profile of pulses for expression (4.5) for $v=0.04, \sigma=0.001$, and $\gamma_{0}=0.01$.

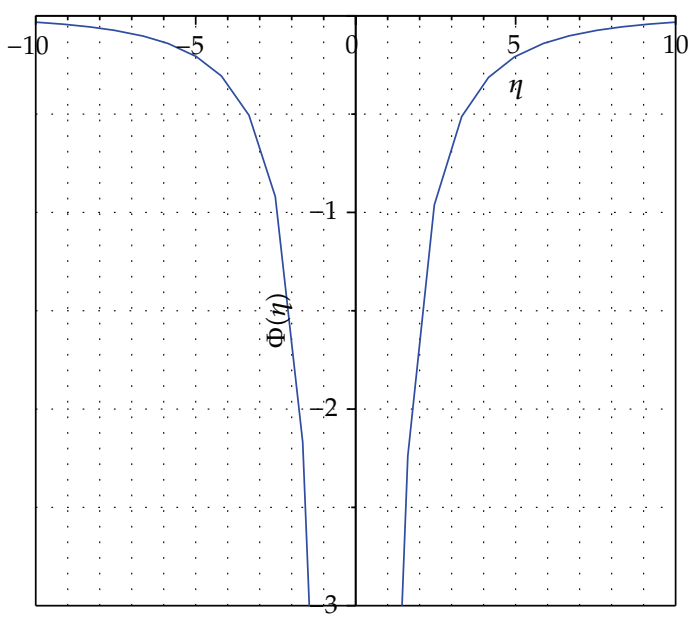

$a_{0}=0$

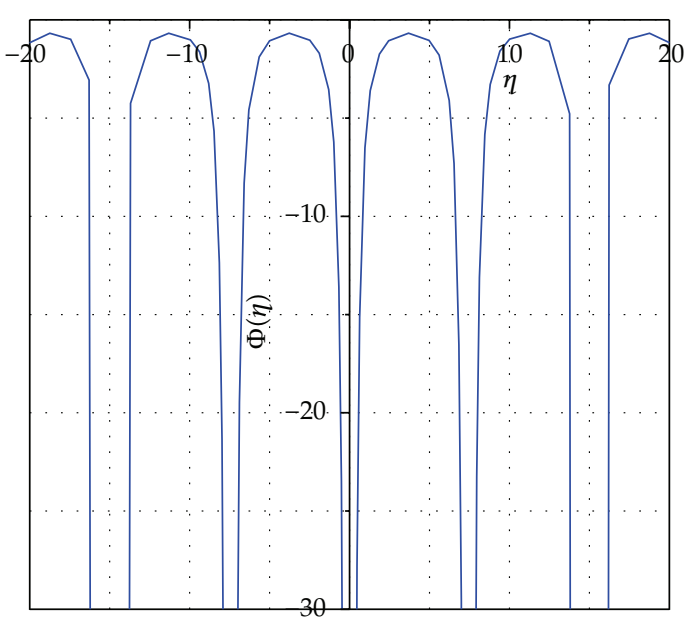

$a_{0}=0.4$

(a)

(b)

Figure 3: The explosive solution for expression (4.7) for $v=0.04, \sigma=0.001$, and $\gamma_{0}=0.01$.

Washimi and Taniuti [7] were the first to use reductive perturbation method to study the propagation of a slow modulation of quasi-monochromatic waves through plasma. And then the attention has been focused by many authors [8-11].

The evolution of small-but finite-amplitude solitary waves, studied by means of the Korteweg-de Vries (KdV) equation, is of considerable interest in plasma dynamics.

Many powerful methods have been established and developed to study nonlinear evolution equations (NLEEs). These methods include the inverse scatting method [12], 


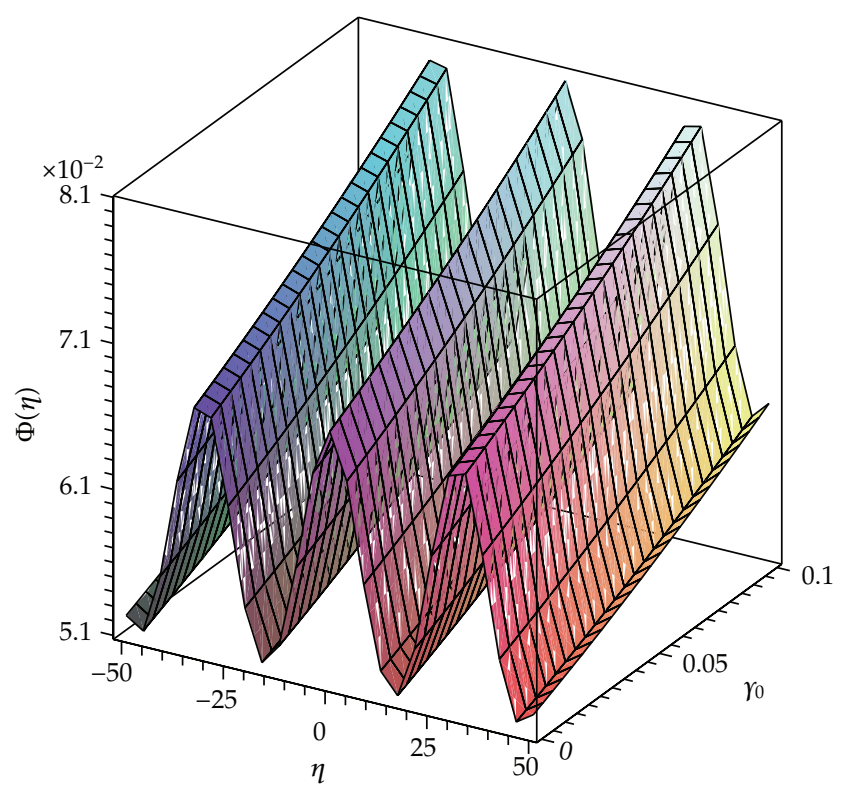

Figure 4: The asymptotical property of Jacobi elliptic doubly periodic wave for expression (4.10) with $v=0.04, \sigma=0.001, a_{0}=0$, and $m=2$.

the tanh function method [13], extended tanh method [14-16], the Exp-function method [17], the extended F-expansion method [18], the Jacobi elliptic function expansion method [19], the homogeneous balance method [20], sech-function method [21], F-expansion method [22], and the multiple exp-function method [23]. There is no unified method that can be used to deal with all types of NLEEs. Fan [24] developed a new algebraic method with computerized symbolic computation, which greatly exceeds the applicability of the existing tanh, extended tanh methods, and Jacobi function expansion method in obtaining a series of exact solutions of nonlinear differential equations.

Recently, the ion-acoustic solitary wave in collisionless unmagnetized plasma consisting of warm ions fluid and isothermal electrons is studied using the time-fractional $\mathrm{KdV}$ equation by El-Wakil et al. [25]. They showed that the time fractional can be used to modulate the electrostatic potential wave.

The major topic of this work is to study the ion-acoustic solitary and other type waves in relativistic warm plasma. This paper is organized as follows. In Section 2, we present the basic set of fluid equations governing our plasma model. In Section 3, an algorithm describing the computerized symbolic computation method is presented. In Section 4, explicit solutions for $\mathrm{KdV}$ equation are obtained. Finally, some discussions and conclusions are given in Section 5.

\section{Basic Equations and KdV Equation}

Consider collisionless ionization-free unmagnetized plasma consisting of a mixture of warm ion-fluid and isothermal electrons. Assume that the ion flow velocity has a weak relativistic effect, and therefore there exist streaming ions in an equilibrium state when sufficiently 


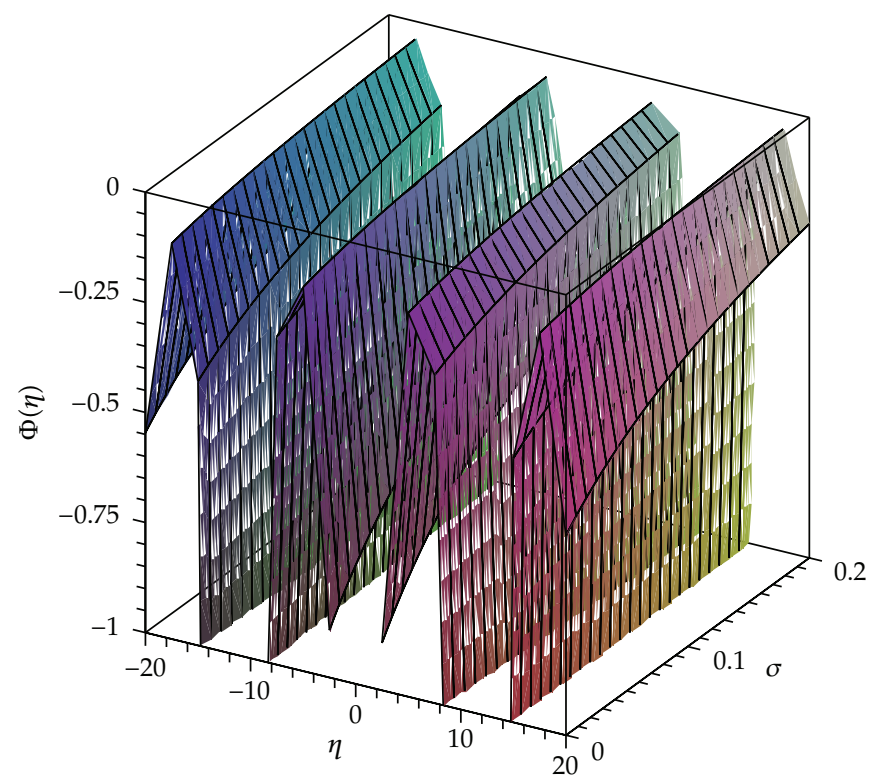

Figure 5: Weierstrass elliptic doubly periodic type solution (4.13) for $v=0.04, \gamma_{0}=0.01, c_{0}=-1, c_{1}=1$, and $c_{3}=0.1$.

small- but finite-amplitude waves propagate one-dimensionally. Such a system is governed by the following normalized equations [26]:

$$
\begin{gathered}
\frac{\partial}{\partial t} n(x, t)+\frac{\partial}{\partial x}[n(x, t) u(x, t)]=0, \\
{\left[\frac{\partial}{\partial t}+u(x, t) \frac{\partial}{\partial x}\right][\gamma(x, t) u(x, t)]+\frac{\sigma}{n(x, t)} \frac{\partial}{\partial x} p(x, t)+\frac{\partial}{\partial x} \phi(x, t)=0,} \\
{\left[\frac{\partial}{\partial t}+u(x, t) \frac{\partial}{\partial x}\right] p(x, t)+3 p(x, t) \frac{\partial}{\partial x}[\gamma(x, t) u(x, t)]=0,} \\
\frac{\partial^{2}}{\partial x^{2}} \phi(x, t)+n(x, t)-n_{e}(x, t)=0 .
\end{gathered}
$$

The electron temperature $T_{e}$ is much larger than the ion temperature $T_{i}$ and in this case for simplicity one can neglect the inertia of the electrons relative to that of the ions, that is, the high-frequency plasma oscillations are neglected. Since it is interested with the regime of density and velocity fluctuations near the ion plasma frequency, so the isothermal electrons density is given by

$$
n_{e}(x, t)=\exp [\phi(x, t)]
$$


For weakly relativistic effects, the relativistic factor $\gamma(x, t)=1 / \sqrt{1-u^{2}(x, t) / c^{2}}$ is approximated by

$$
r(x, t) \approx 1+\frac{u^{2}(x, t)}{\left(2 c^{2}\right)}
$$

where $c$ is the velocity of light.

In (2.1a) $-(2.1 \mathrm{f}), n(x, t)$ and $n_{e}(x, t)$ are the densities of ions and electrons, respectively, $u(x, t)$ is the ion flow velocity, $p(x, t)$ is the ion pressure, $\phi(x, t)$ is the electric potential, $x$ is the space coordinate, and $t$ is the time variable. $\sigma=T_{i} / T_{e} \ll 1$ is the ratio of the ion temperature to the electron temperature. All of these quantities are dimensionless and are normalized in terms of the following characteristic quantities: $n(x, t)$ and $n_{e}(x, t)$ by the unperturbed electron density $n_{0}, u(x, t)$ and $c$ by the sound velocity $\sqrt{k_{B} T_{e} / m_{i}}, p(x, t)$ and $\phi(x, t)$ by $n_{0} k_{B} T_{i}$ and $k_{B} T_{e} / e$, respectively; $t$ and $x$ by the inverse of the plasma frequency $\omega_{p i}^{-1}=1 / \sqrt{4 \pi e^{2} n_{0} / m_{i}}$ and the electron Debye length $\lambda_{D}=\sqrt{k_{B} T_{e} /\left(4 \pi e^{2} n_{0}\right)}$, respectively, $k_{B}$ is Boltzmann's constant, and $m_{i}$ is the mass of plasma ion.

According to the general method of reductive perturbation theory, the stretched variables are introduced as [7]

$$
\tau=\varepsilon^{3 / 2} t, \quad \xi=\varepsilon^{1 / 2}(x-\lambda t),
$$

where $\lambda$ is the phase velocity and $\varepsilon$ represents the amplitude of the perturbation. All the physical quantities that appeared in (2.1a)-(2.1f) are expanded as power series in $\varepsilon$ about the equilibrium values as

$$
\begin{gathered}
n(\xi, \tau)=1+\varepsilon n_{1}(\xi, \tau)+\varepsilon^{2} n_{2}(\xi, \tau)+\cdots, \\
u(\xi, \tau)=u_{0}+\varepsilon u_{1}(\xi, \tau)+\varepsilon^{2} u_{2}(\xi, \tau)+\cdots, \\
p(\xi, \tau)=1+\varepsilon p_{1}(\xi, \tau)+\varepsilon^{2} p_{2}(\xi, \tau)+\cdots, \\
\phi(\xi, \tau)=\varepsilon \phi_{1}(\xi, \tau)+\varepsilon^{2} \phi_{2}(\xi, \tau)+\cdots .
\end{gathered}
$$

The boundary conditions of this problem are imposed as $|\xi| \rightarrow \infty, n=n_{e}=p=1, u=u_{0}$, and $\phi=0$.

Substituting (2.2) and (2.3a)-(2.3d) into the system of (2.1a)-(2.1f) and equating the coefficients of like powers of $\varepsilon$, then from the lowest order

$$
\begin{gathered}
n_{1}(\xi, \tau)=\phi_{1}(\xi, \tau), \\
u_{1}(\xi, \tau)=\left(\lambda-u_{0}\right) \phi_{1}(\xi, \tau), \\
p_{1}(\xi, \tau)=3 \gamma_{1} \phi_{1}(\xi, \tau),
\end{gathered}
$$


with the transcendental equation of $\lambda$ that is given as

$$
1-\left(\lambda^{2}-3 \sigma\right) \gamma_{1}=0 \Longrightarrow \lambda= \pm \sqrt{\frac{\left(3 \sigma \gamma_{1}+1\right)}{\gamma_{1}}}
$$

where

$$
r_{1}=1+\frac{3 \gamma_{0}^{2}}{2}, \quad r_{0}=\frac{u_{0}}{c}
$$

Using second-order equations in $\varepsilon$ and eliminating the second-order perturbed quantities $n_{2}$, $u_{2}, p_{2}$, and $\phi_{2}$, the following $\mathrm{KdV}$ equation for the first-order perturbed potential is obtained:

$$
\frac{\partial}{\partial \tau} \phi_{1}(\xi, \tau)+A \phi_{1}(\xi, \tau) \frac{\partial}{\partial \xi} \phi_{1}(\xi, \tau)+B \frac{\partial^{3}}{\partial \xi^{3}} \phi_{1}(\xi, \tau)=0
$$

Therefore, the nonlinear coefficient $A$ and the dispersion coefficient $B$ are represented by

$$
A=B\left(g_{1}-\frac{g_{2} \gamma_{2}}{\sqrt{\gamma_{1}^{3}}}\right), \quad B=\frac{1}{g_{2} \sqrt{\gamma_{1}}}
$$

with

$$
g_{1}=3 \sigma \gamma_{1}\left(3 \gamma_{1}+1\right)+2, \quad g_{2}=2 \sqrt{3 \sigma \gamma_{1}+1}, \quad \gamma_{2}=\frac{3 \gamma_{0}}{(2 c)} .
$$

\section{Computerized Symbolic Computation Method}

An algebraic method with computerized symbolic computation has been developed by Fan [24], which can be used to solve a given partial differential equation in $\phi_{1}(\xi, \tau)$ of the form

$$
H\left(\phi_{1}, \frac{\partial \phi_{1}}{\partial \tau}, \frac{\partial \phi_{1}}{\partial \xi}, \frac{\partial^{2} \phi_{1}}{\partial \xi^{2}}, \ldots\right)=0 .
$$

This equation may be transformed into an ordinary differential equation of the form

$$
H\left(\Phi, \frac{d \Phi}{d \eta}, \frac{d^{2} \Phi}{d \eta^{2}}, \ldots\right)=0
$$

using a traveling frame of reference

$$
\phi_{1}(\xi, \tau)=\Phi(\eta), \quad \eta=\xi-v \tau,
$$


where $v$ is the traveling wave propagation velocity.

The computational technique used to solve the NLEE is described as follows [24].

Step 1. Reduce partial differential equation (3.1a) to the ordinary differential equation (3.1b) by considering the traveling wave transformation (3.1c).

Step 2. Expand the solution of $(3.1 \mathrm{~b})$ in the form

$$
\Phi(\eta)=\sum_{i=0}^{n} a_{i} \varphi^{i}(\eta)
$$

where the new variable $\varphi(\eta)$ is a solution of the following ordinary differential equation:

$$
\frac{d \varphi(\eta)}{d \eta}=\in \sqrt{\sum_{j=0}^{r} c_{j} \varphi^{j}(\eta)}, \quad \in= \pm 1
$$

Step 3. Substituting (3.2b) into (3.1b) and balancing the highest derivative term with the highest nonlinear term lead to a relation between $n$ and $r$, from which the different possible values of $n$ and $r$ can be obtained. These values lead to the different series expansions of the solutions.

Step 4. Substituting the expansions (3.2a)-(3.2b) into (3.1b) and setting the coefficients of all powers of $\varphi^{i}$ and $\varphi^{i} d \varphi / d \eta$ to zero will give a system of algebraic equations, from which the parameters $a_{i}(i=0,1, \ldots, n)$ and $c_{j}(j=0,1, \ldots, r)$ can be found explicitly.

Step 5. Substituting the parameters $c_{j}(j=0,1, \ldots, r)$ obtained in Step 4 into (3.2b) gives all the possible solutions $\varphi(\eta)$.

It is remarked here that the solutions of (3.1a) depend on the explicit solvability of (3.2b). The solutions of (3.2b) will get a series of fundamental solutions such as polynomial, exponential, soliton, rational, triangular periodic, Jacobi, and Weierstrass elliptic doubly periodic solutions. 


\section{Explicit Solutions for the KdV Equation}

For KdV equation (2.5a), the traveling wave transformation (3.1c) leads to

$$
-v \frac{d}{d \eta} \Phi(\eta)+A \Phi(\eta) \frac{d}{d \eta} \Phi(\eta)+B \frac{d^{3}}{d \eta^{3}} \Phi(\eta)=0
$$

Balancing the highest derivative term with the highest nonlinear term leads to a relation between $n$ and $r$ as $r=n+2$. Taking $n=2$ gives $r=4$ and leads to

$$
\begin{gathered}
\Phi(\eta)=a_{0}+a_{1} \varphi(\eta)+a_{2} \varphi^{2}(\eta) \\
\frac{d \varphi}{d \eta}=\in \sqrt{c_{0}+c_{1} \varphi(\eta)+c_{2} \varphi^{2}(\eta)+c_{3} \varphi^{3}(\eta)+c_{4} \varphi^{4}(\eta)} .
\end{gathered}
$$

Substituting (4.2a)-(4.2b) into (4.1) and equating coefficients of all powers of $\varphi^{i}$ and $\varphi^{i} d \varphi / d \eta$ to zero will get a system of algebraic equations, from which the parameters $a_{i}(i=0,1,2)$ and $c_{j}(j=0,1, \ldots, 4)$ can be found explicitly. Substituting $c_{j}$ into $(4.2 \mathrm{~b})$ and using the symbolic software package Maple give explicit solutions of (4.2b).

Substituting the coefficients $a_{i}$ and (4.2a) into (4.1) and using the symbolic software package Maple, we obtain, for KdV equation (4.1), the following solutions:

$$
\begin{gathered}
\Phi(\eta)=a_{0}-3 \frac{\left(A a_{0}-v\right)}{A} \operatorname{sech}^{2}\left(\frac{1}{2} \sqrt{\frac{\left(v-A a_{0}\right)}{B}} \eta\right), \quad c_{0}=c_{1}=c_{4}=0, \quad \frac{\left(v-A a_{0}\right)}{B}>0, \\
\Phi(\eta)=a_{0}-3 \frac{\left(A a_{0}-v\right)}{A} \sec ^{2}\left(\frac{1}{2} \sqrt{\frac{\left(A a_{0}-v\right)}{B}} \eta\right), \quad c_{0}=c_{1}=c_{4}=0, \quad \frac{\left(v-A a_{0}\right)}{B}<0, \\
\Phi(\eta)=a_{0}+\frac{3}{2} \frac{\left(A a_{0}-v\right)}{A} \tan ^{2}\left(\frac{1}{2} \sqrt{\frac{\left(v-A a_{0}\right)}{2 B}} \eta\right), \quad c_{0} \neq 0, \\
c_{1}=c_{3}=0, \quad \frac{\left(v-A a_{0}\right)}{B}>0,
\end{gathered}
$$




$$
\begin{aligned}
& \Phi(\eta)=a_{0}-\frac{3}{2} \frac{\left(A a_{0}-v\right)}{A} \tanh ^{2}\left(\frac{1}{2} \sqrt{\frac{\left(A a_{0}-v\right)}{2 B} \eta}\right), \quad c_{0} \neq 0, \\
& c_{1}=c_{3}=0, \quad \frac{\left(v-A a_{0}\right)}{B}<0, \\
& \Phi(\eta)=a_{0}+3 \frac{\left(A a_{0}-v\right)}{A} \operatorname{csch}^{2}\left(\frac{1}{2} \sqrt{\frac{\left(v-A a_{0}\right)}{B} \eta}\right) \\
& c_{0}=c_{1}=c_{3}=0, \quad \frac{\left(v-A a_{0}\right)}{B}>0, \\
& \Phi(\eta)=a_{0}-3 \frac{\left(A a_{0}-v\right)}{A} \csc ^{2}\left(\frac{1}{2} \sqrt{\frac{\left(A a_{0}-v\right)}{B} \eta}\right) \\
& c_{0}=c_{1}=c_{3}=0, \quad \frac{\left(v-A a_{0}\right)}{B}<0, \\
& \Phi(\eta)=\frac{v}{A}-\frac{12 B}{A \eta^{2}}, \quad c_{0}=c_{1}=c_{3}=0, \quad \frac{\left(v-A a_{0}\right)}{B}=0, \\
& \Phi(\eta)=a_{0}-3 \frac{\left(A a_{0}-v\right)}{A} \frac{m^{2}}{\left(2 m^{2}-1\right)} \mathrm{cn}^{2}\left(\frac{1}{2} \sqrt{\frac{A a_{0}-v}{B\left(1-2 m^{2}\right)} \eta}\right), \quad c_{0} \neq 0, \\
& c_{1}=c_{3}=0, \quad \frac{v-a_{0} A}{B}>0, \\
& \Phi(\eta)=a_{0}+3 \frac{\left(A a_{0}-v\right)}{A} \frac{1}{\left(m^{2}-2\right)} \mathrm{dn}^{2}\left(\frac{1}{2} \sqrt{\frac{A a_{0}-v}{B\left(m^{2}-2\right)} \eta}\right), \quad c_{0} \neq 0, \\
& c_{1}=c_{3}=0, \quad \frac{v-a_{0} A}{B}>0, \\
& \Phi(\eta)=a_{0}-3 \frac{\left(A a_{0}-v\right)}{A} \frac{m^{2}}{\left(m^{2}+1\right)} \operatorname{sn}^{2}\left(\frac{1}{2} \sqrt{\frac{A a_{0}-v}{B\left(m^{2}+1\right)} \eta}\right), \quad c_{0} \neq 0, \\
& c_{1}=c_{3}=0, \quad \frac{v-a_{0} A}{B}<0, \\
& \Phi(\eta)=\frac{v}{A}-3 \sqrt[3]{4 c_{3}^{2}} \frac{B}{A} \wp\left(\sqrt[3]{\frac{c_{3}}{4}} \eta ;-\sqrt[3]{\frac{4}{c_{3}}} c_{1},-c_{0}\right), \quad c_{2}=c_{4}=0, \quad c_{3}>0
\end{aligned}
$$

where $m$ is the modulus of the Jacobi elliptic functions, $a_{0}, c_{0}, c_{1}$, and $c_{3}$ are arbitrary constants, and $\wp$ is the Weierstrass elliptic doubly periodic function.

\section{Results and Discussion}

Solutions (4.3) and (4.6) are hyperbolic wave solutions. In Figure 1, a profile of the bellshaped solitary pulse is obtained for solution (4.3). Figures (1(a) and $1(\mathrm{~b})$ ) show that the 
soliton amplitude and width are sensitive to the relativistic factor $\gamma_{0}$ and the temperatures ratio $\sigma$. Also, the arbitrary value $a_{0}$ gives the same effect of adding a higher-order perturbation correction in increasing the amplitude and decreasing the width as shown in Figure 1(c).

Solutions (4.4) and (4.5) are triangular solutions that develop solitons with singularity at finite points, which are called "blowup" solutions [27] as in Figure 2(a). In Figure 2(b), for some values of $a_{0}$ the pulse does not vanish to infinity, so it has a localized form. Therefore, the arbitrary value $a_{0}$ plays a role in obtaining a pulse-shaped localized solution [28]. Solutions (4.7) and (4.8) lead to the propagation of an explosive pulses [29]. In Figure 3, the profiles of explosive (divergent) pulses are depicted for expression (4.7). For $a_{0}=0$, the solution gives a localized explosive pulse, as in Figure (2.3a) while a periodic solution is obtained for $a_{0}=0.4$, as shown in Figure (2.3b). The rational solution (4.9) may be helpful to explain certain physical phenomena. Because a rational solution is a disjoint union of manifolds, particle systems describing the motion of a pole of rational solutions for a $\mathrm{KdV}$ equation were analyzed [30]. Equations (4.10)-(4.12) are three Jacobi elliptic doubly periodic wave solutions. When $m \rightarrow 1$, solutions (4.10) and (4.11) reduce to (4.3) while (4.11) reduces to (4.6) [31]. In Figure 4, a profile of triangular periodic wave solution for expression (4.10) for $m=2$ is shown. On the other hand, (4.13) gives the Weierstrass elliptic doubly periodic type solution as depicted in Figure 5.

In summary, it has been found that the amplitude and the width of the ion-acoustic waves as well as parametric regime where the solitons can exist are sensitive to the relativistic factor $\gamma_{0}$ and the ratio of the ion to the electron temperatures $\sigma$. Moreover, solutions for KdV equation have been obtained. It may be important to explain some physical phenomena in some plasma environments, such as ionosphere region.

\section{References}

[1] G. B. Whitham, Linear and Nonlinear Waves, John Wiley \& Sons, New York, NY, USA, 1974, Pure and Applied Mathematics.

[2] R. Davidson, Methods in Nonlinear Plasma Theory, Academic Press, New York, NY, USA, 1972.

[3] G. C. Das and S. N. Paul, "Ion-acoustic solitary waves in relativistic plasmas," Physics of Fluids, vol. 28, no. 3, pp. 823-825, 1985.

[4] Y. Nejoh, "The effect of the ion temperature on the ion acoustic solitary waves in a collisionless relativistic plasma," Journal of Plasma Physics, vol. 37, no. 3, pp. 487-495, 1987.

[5] Y. Nejoh, "A two-dimensional ion acoustic solitary wave in a weakly relativistic plasma," Journal of Plasma Physics, vol. 38, no. 3, pp. 439-444, 1987.

[6] S. K. El-Labany, "Contribution of higher-order nonlinearity to nonlinear ion-acoustic waves in a weakly relativistic plasma," Journal of Plasma Physics, vol. 50, no. 3, pp. 495-504, 1993.

[7] H. Washimi and T. Taniuti, "Propagation of ion-acoustic solitary waves of small amplitude," Physical Review Letters, vol. 17, no. 19, pp. 996-998, 1966.

[8] T. Taniuti and C. C. Wei, "Reductive perturbation method in nonlinear wave propagation. I," Journal of the Physical Society of Japan, vol. 24, no. 4, pp. 941-946, 1968.

[9] T. Taniuti, "Reductive perturbation method and far fields of wave equations," Progress of Theoretical Physics Supplement, no. 55, pp. 1-35, 1974.

[10] V. I. Karpman, Non-Linear Waves in Dispersive Media, Pergamon Press, Oxford, UK, 1975.

[11] E. K. El-Shewy, "Effect of higher-order nonlinearity to nonlinear electron-acoustic solitary waves in an unmagnetized collisionless plasma," Chaos, Solitons and Fractals, vol. 26, no. 4, pp. 1073-1079, 2005.

[12] M. J. Ablowitz and P. A. Clarkson, Solitons, Nonlinear Evolution Equations and Inverse Scattering, vol. 149 of London Mathematical Society Lecture Note Series, Cambridge University Press, Cambridge, UK, 1991.

[13] E. J. Parkes and B. R. Duffy, “An automated tanh-function method for finding solitary wave solutions to non-linear evolution equations," Computer Physics Communications, vol. 98, no. 3, pp. 288-300, 1996. 
[14] E. Fan, "Extended tanh-function method and its applications to nonlinear equations," Physics Letters A, vol. 277, no. 4-5, pp. 212-218, 2000.

[15] S. A. Elwakil, S. K. El-Labany, M. A. Zahran, and R. Sabry, "Modified extended tanh-function method for solving nonlinear partial differential equations," Physics Letters A, vol. 299, no. 2-3, pp. 179-188, 2002.

[16] E. Yusufoğlu and A. Bekir, "On the extended tanh method applications of nonlinear equations," International Journal of Nonlinear Science, vol. 4, no. 1, pp. 10-16, 2007.

[17] J.-H. He and M. A. Abdou, "New periodic solutions for nonlinear evolution equations using Expfunction method," Chaos, Solitons and Fractals, vol. 34, no. 5, pp. 1421-1429, 2007.

[18] M.-L. Wang and X.-Z. Li, "Extended F-expansion method and periodic wave solutions for the generalized Zakharov equations," Physics Letters A, vol. 343, no. 1-3, pp. 48-54, 2005.

[19] M. A. Abdou and A. Elhanbaly, "Construction of periodic and solitary wave solutions by the extended Jacobi elliptic function expansion method," Communications in Nonlinear Science and Numerical Simulation, vol. 12, no. 7, pp. 1229-1241, 2007.

[20] M.-L. Wang, "Solitary wave solutions for variant Boussinesq equations," Physics Letters A, vol. 199, no. 3-4, pp. 169-172, 1995.

[21] W.-X. Ma, "Travelling wave solutions to a seventh order generalized KdV equation," Physics Letters A, vol. 180, no. 3, pp. 221-224, 1993.

[22] Y. Zhou, M.-L. Wang, and Y.-M. Wang, "Periodic wave solutions to a coupled KdV equations with variable coefficients," Physics Letters A, vol. 308, no. 1, pp. 31-36, 2003.

[23] W.-X. Ma, T.-W. Huang, and Y. Zhang, "A multiple exp-function method for nonlinear differential equations and its application," Physica Scripta, vol. 82, no. 6, Article ID 065003, 8 pages, 2010.

[24] E. Fan, "A new algebraic method for finding the line soliton solutions and doubly periodic wave solution to a two-dimensional perturbed KdV equation," Chaos, Solitons and Fractals, vol. 15, no. 3, pp. 567-574, 2003.

[25] S. A. El-Wakil, E. M. Abulwafa, E. K. El-Shewy, and A. A. Mahmoud, "Ion-acoustic waves in plasma of warm ions and isothermal electrons using time-fractional KdV equation," Chinese Physics B, vol. 20, no. 4, Article ID 040508, 2011.

[26] S. K. El-Labany and A. M. El-Hanbaly, "Modulation of the non-linear ion acoustic waves in a plasma consisting of warm ions and isothermal electrons," IL Nuovo Cimento D, vol. 17, no. 6, pp. 547-556, 1995.

[27] W. M. Moslem, R. Sabry, U. M. Abdelsalam, I. Kourakis, and P. K. Shukla, "Solitary and blow-up electrostatic excitations in rotating magnetized electron-positron-ion plasmas," New Journal of Physics, vol. 11, no. 3, Article ID 033028, 2009.

[28] R. Sabry, W. M. Moslem, and P. K. Shukla, "Explosive and solitary excitations in a very dense magnetoplasma," Physics Letters A, vol. 372, no. 35, pp. 5691-5694, 2008.

[29] M. Adler and J. Moser, "On a class of polynomials connected with the Korteweg-de Vries equation," Communications in Mathematical Physics, vol. 61, no. 1, pp. 1-30, 1978.

[30] D. Baldwin, Ü. Göktaş, W. Hereman, L. Hong, R. S. Martino, and J. C. Miller, "Symbolic computation of exact solutions expressible in hyperbolic and elliptic functions for nonlinear PDEs," Journal of Symbolic Computation, vol. 37, no. 6, pp. 669-705, 2004.

[31] E. K. El-Shewy, H. G. Abdelwahed, and H. M. Abd-El-Hamid, "Computational solutions for the Korteweg-deVries equation in warm plasma," Computational Methods in Science and Technology, vol. 16, no. 1, pp. 13-18, 2010. 


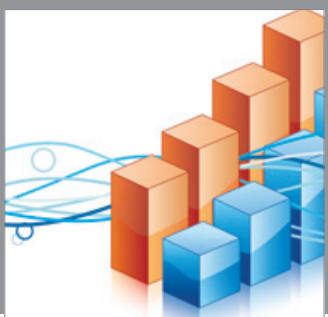

Advances in

Operations Research

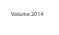

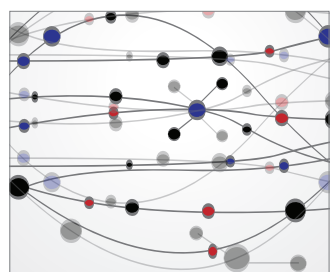

\section{The Scientific} World Journal
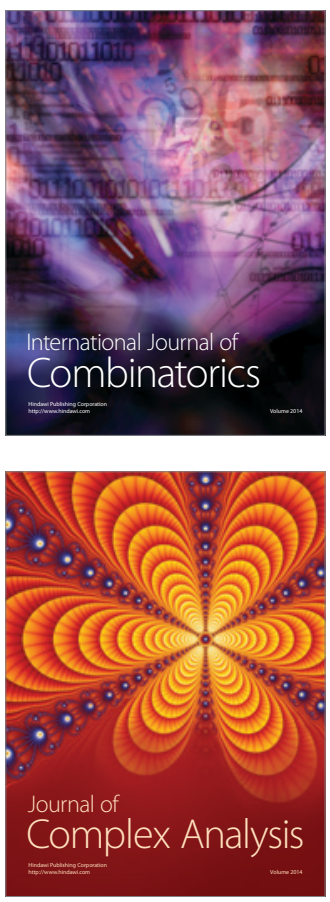

International Journal of

Mathematics and

Mathematical

Sciences
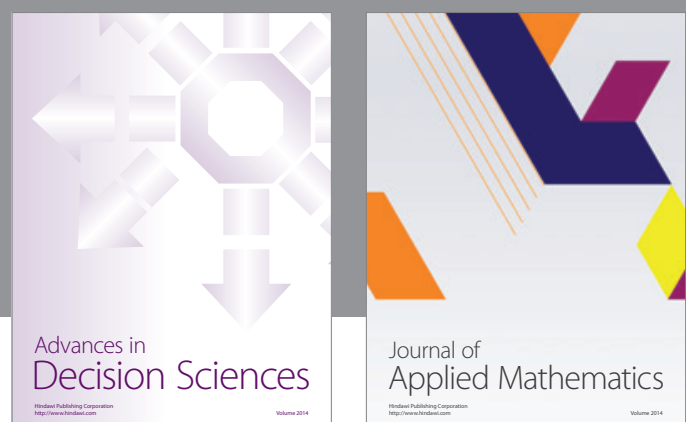

Journal of

Applied Mathematics
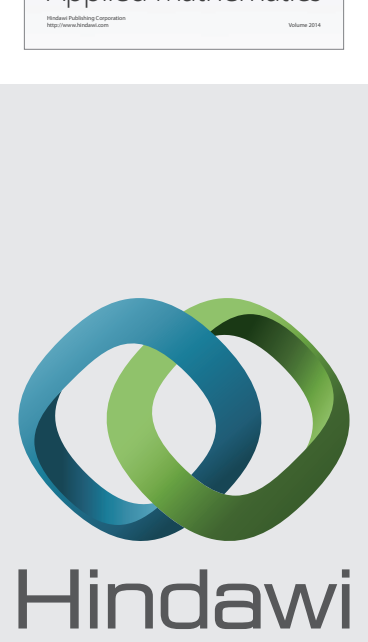

Submit your manuscripts at http://www.hindawi.com
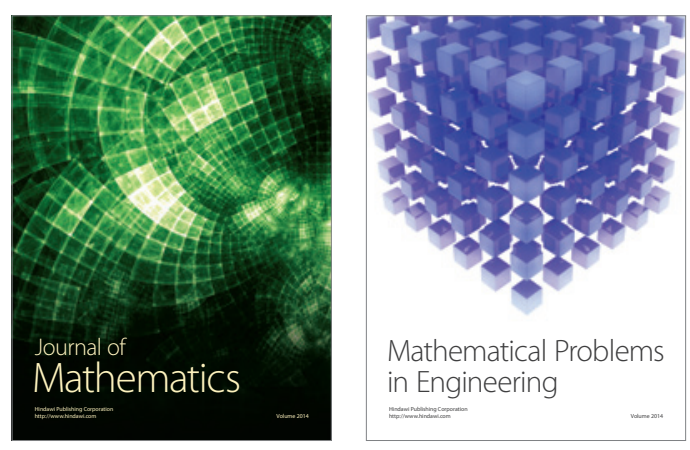

Mathematical Problems in Engineering
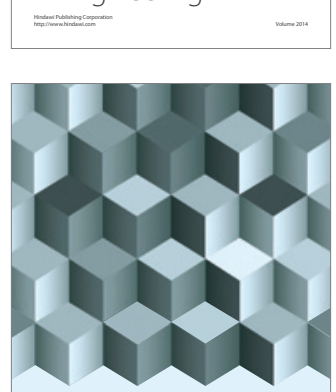

Journal of

Function Spaces
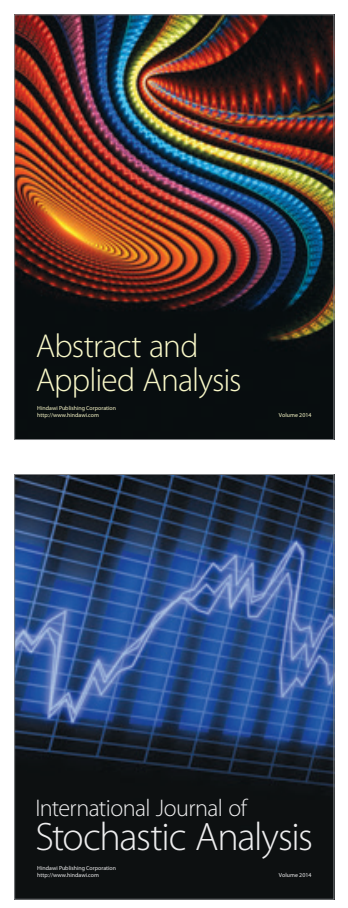

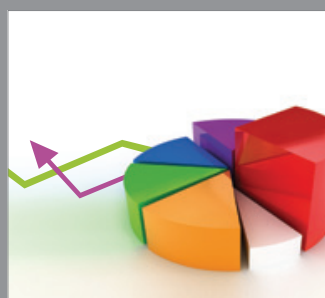

ournal of

Probability and Statistics

Promensencen
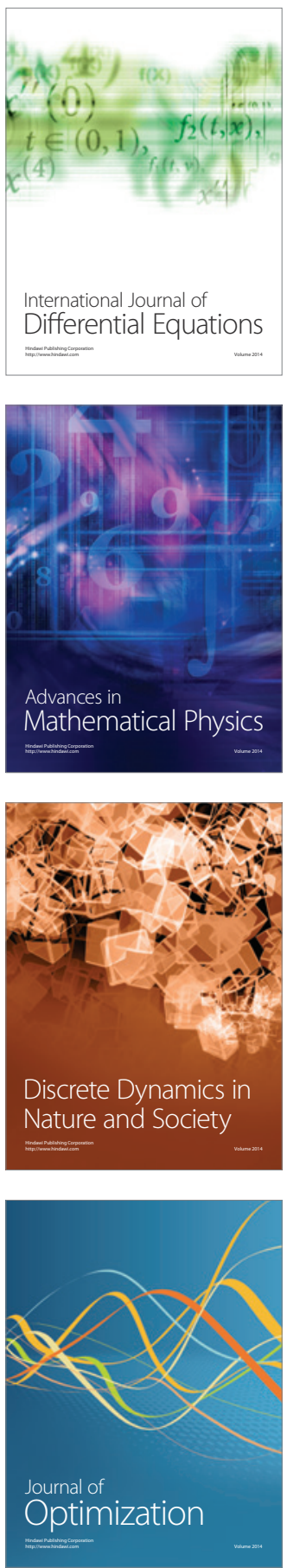